\title{
Biosafety Level
}

National Cancer Institute

\section{Source}

National Cancer Institute. Biosafety Level. NCI Thesaurus. Code C151920.

A set of biocontainment precautions, consisting of combinations of laboratory practices and techniques, safety equipment, and specialized laboratory facilities, required to isolate potentially dang erous biological ag ents in an enclosed laboratory facility. They include individual safeguards designed to protect laboratory personnel, as well as safeguards designed to protect the surrounding environment and community. 